\title{
A single mutation in the ACTR8 gene associated with lineage-specific expression in primates
}

Se-Hee Choe ${ }^{1,2 \dagger}$, Sang-Je Park ${ }^{1 \dagger}$, Hyeon-Mu Cho ${ }^{1,2 \dagger}$, Hye-Ri Park ${ }^{1,2}$, Ja-Rang Lee ${ }^{3}$, Young-Hyun Kim ${ }^{1,2^{*}}$ and Jae-Won Huh ${ }^{1,2^{*}}$ (D)

\begin{abstract}
Background: Alternative splicing (AS) generates various transcripts from a single gene and thus plays a significant role in transcriptomic diversity and proteomic complexity. Alu elements are primate-specific transposable elements (TEs) and can provide a donor or acceptor site for AS. In a study on TE-mediated AS, we recently identified a novel AluSz6-exonized ACTR8 transcript of the crab-eating monkey (Macaca fascicularis). In the present study, we sought to determine the molecular mechanism of AluSz6 exonization of the ACTR8 gene and investigate its evolutionary and functional consequences in the crab-eating monkey.

Results: We performed RT-PCR and genomic PCR to analyze AluSz6 exonization in the ACTR8 gene and the expression of the AluSz6-exonized transcript in nine primate samples, including prosimians, New world monkeys, Old world monkeys, and hominoids. AluSz6 integration was estimated to have occurred before the divergence of simians and prosimians. The Alu-exonized transcript obtained by AS was lineage-specific and expressed only in Old world monkeys and apes, and humans. This lineage-specific expression was caused by a single G duplication in AluSz6, which provides a new canonical 5' splicing site. We further identified other alternative transcripts that were unaffected by the AluSz6 insertion. Finally, we observed that the alternative transcripts were transcribed into new isoforms with C-terminus deletion, and in silico analysis showed that these isoforms do not have a destructive function.
\end{abstract}

Conclusions: The single G duplication in the TE sequence is the source of TE exonization and AS, and this mutation may suffer a different fate of ACTR8 gene expression during primate evolution.

Keywords: Alternative splicing, Alu, ACTR8, Exonization, Lineage-specific expression

\section{Background}

Alternative splicing (AS) is a molecular mechanism producing various transcripts and diverse proteins from a single gene and plays an important role in genomic and phenotypic complexity $[1,2]$. In the human genome,

\footnotetext{
* Correspondence: kyh@kribb.re.kr; huhjw@kribb.re.kr

'Se-Hee Choe, Sang-Je Park and Hyeon-Mu Cho contributed equally to this work.

${ }^{1}$ National Primate Research Center, Korea Research Institute of Bioscience and Biotechnology (KRIBB), Cheongju 28116, Korea

Full list of author information is available at the end of the article
}

more than $95 \%$ of pre-mRNAs undergo AS $[3,4]$. AS mechanisms are classified into five types, including exon skipping, alternative $3^{\prime}$ splice site (SS), alternative $5^{\prime} \mathrm{SS}$, intron retention, and mutual exclusion [5, 6]. Exon creation by duplication and mutation within repetitive elements has been reported to be highly relevant to AS and to impact mammalian evolution [7-10]. A transcriptome study of the crab-eating monkey (Macaca fascicularis) reported that $10 \%$ of AS events were associated with TEs [11]. 
TEs are mobile genetic elements that can change their position in the genome and thus can affect the sequence and structure of genes. Accordingly, they can modulate gene functions in a relatively short time and are considered as an evolutionary driving force $[12,13]$. They constitute over $45 \%$ of the human genome and are categorized into two classes. Class I TEs, known as retrotransposons, require reverse transcription for their activation, and class II TEs of DNA transposons encode transposases responsible for their excision and insertion [14-16]. Retrotransposons comprise approximately $42 \%$ of the human genome and include endogenous retroviruses (ERVs); long interspersed elements (LINEs); short interspersed elements (SINEs), as well as SINE-R; variable number of tandem repeats (VNTRs); and Alu elements (known as SVA elements for SINE/VNTR/Alu) $[17,18]$. Alu and SINE elements are the most successful of all TEs in the primate genome. Alu elements are slightly less than 300 base pairs (bp) in length, present at over than 1 million copies, and widely dispersed within introns and genes in the human genome [19]. Alu elements generally consist of distinct monomeric left and right arms, an A-rich linker, and a poly(A) tail [19]. These features facilitate the regulation of gene expression by alternative polyadenylation and non-allelic Alu/ Alu recombination, causing genomic instability and eventually contributing to primate genome divergence [20-22]. In addition, when the antisense-oriented Alu integrates into a gene, the poly(A) tails could serve as a polypyrimidine tract (PPT) for the recognition of the cryptic splice site. Thus, Alu elements are a major source of cryptic exons through exonization [23]. Alu elements affect gene function through the generation of different splicing isoforms. Consequently, Alu exonization contributes to novel functions and genome evolution in primates [20,24].

Arp8 actin-related protein 8 homolog (ACTR8) is a member of the actin superfamily and has $15-72 \%$ sequence identity with actin, which is structurally and evolutionarily similar [25]. As actin-related proteins (Arps) contain the ATP-binding pocket, termed the actin fold, their functions are distinct from those of actin [26]. Unlike actin, Arps are predominantly located in the nucleus and have been associated with nucleosome remodeling, histone acetylation, histone variant exchange, transcription regulation, and DNA repair [27-29]. ACTR8 is a key component of the INO80 complex, which has critical functions in DNA replication, repair, and recombination, as well as in transcription and heterochromatin maintenance [30, 31]. ACTR8 is involved in the ATPase activity of the INO80 complex and recruits the complex to DNA damage sites, and mutation or deletion of ACTR8 has effects similar to INO80 deletion [32]. Furthermore, ACTR8 is involved in transcriptional activation by regulating promoter architecture and contributes to the regulation of cellular function via cytoskeleton organization [33]. Despite numerous functional studies on ACTR8, information on the alternative transcripts and evolution of the ACTR8 gene is limited.

In a study on TE-mediated AS, we recently identified a novel AluSz6-exonized ACTR8 transcript in the crabeating monkey [11]. Therefore, this study aimed to present a series of events of AluSz6 insertion within the ACTR8 gene in the crab-eating monkey and explain the roles of $A l u$ elements in primate evolution.

\section{Results}

Structural analysis of the ACTR8 gene in various primates We conducted a structural analysis of the ACTR8 gene in nine primates, including hominoids (human, chimpanzee, and gorilla), Old world monkeys (rhesus monkey, crab-eating monkey, and African green monkey), New world monkeys (marmoset and squirrel monkey), and prosimians (ring-tailed lemur), using DNA and mRNA sequences from the NCBI genome database. Obtained DNA sequences were screened for repetitive elements using the RepeatMasker Program, which revealed that AluSz6 is located in the 7th intron region in antisense orientation (Fig. 1a). The ACTR8 gene is composed of 13 exons. The length of the untranslated region (UTR) differs between species, but the open reading frame (ORF) region is highly conserved and encodes 624 amino acids. Remarkably, the squirrel monkey ACTR 8 gene has 12 exons and encodes a short protein of 616 amino acids. AluSz6-exonized transcripts were not found from the NCBI database throughout the nine primates. Next, we performed genomic PCR to determine the integration time of AluSz6 using genomic DNA samples. Amplicons containing AluSz6 were detected in all the primates studied (Fig. 1b). These results indicated that AluSz6 was integrated into the primate genome before the divergence of simian and prosimian lineages.

\section{Identification of the Alu-derived and alternative splicing variants of the ACTR8 gene}

To confirm the occurrence of AluSz6 exonization of the $A C T R 8$ gene in the crab-eating monkey, reverse transcription (RT) PCR was performed using two validation primer pairs (V1 and V2) (Fig. 2a and Additional file 1: Table 1). The V1 primer pairs were designed to identify transcript variants, and the $\mathrm{V} 2$ primers pairs were used to detect the transcripts containing the Alu-derived exon. In total, seven transcripts were identified in the crab-eating monkey; the V1 primers detected five transcripts, and the V2 primers detected two transcripts (Fig. 2b). Sequence analysis of the transcripts revealed that the variants originated from multiple AS events, including exon skipping, alternative $3^{\prime}$ SS and 5' SS, intron retention, mutual exclusion, 


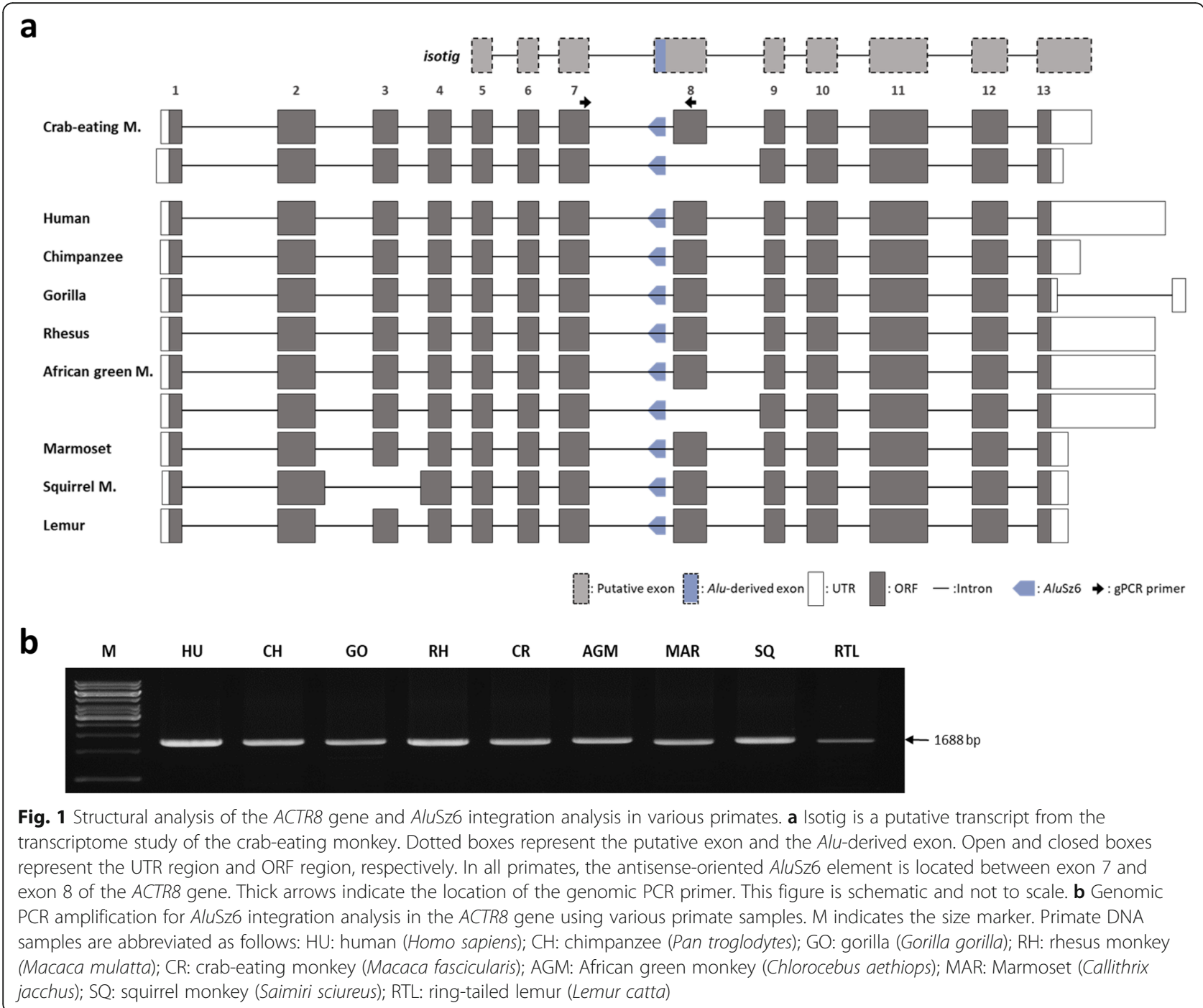

and Alu exonization (Fig. 2c) [5, 6]. The TV1 transcript skips exon 8 and has exon 9a, which is 19 bp longer than exon 9, through the use of alternative 3' SS. The TV2 transcript has exon 7a and an AluSz6-derived exon, which are generated by mutual exclusion and $A l u$ exonization, respectively. TV3 and TV4 have the same AluSz6-derived exon but carry exon 9 and exon 9a, respectively, through differential alternative 3' SS. TV5 is generated by simultaneous AluSz6 exonization and intron retention. TV6 has a longer AluSz6derived exon due to a differential alternative $5^{\prime}$ SS.

Because $A l u$-exonized transcripts often exhibit tissuespecific expression patterns [34-36], we profiled ACTR8 gene expression in various tissues of the crab-eating monkey, including the cerebellum, cerebrum, heart, kidney, lung, pancreas, spleen, and testis. Specific RT-PCR primers for seven transcript variants were designed, considering the splice junctions of each (Fig. 2c). RT-PCR results did not reveal tissue-specific ACTR8 gene expression. The original transcript was ubiquitously expressed in all tissues evaluated, whereas the other transcript variants (TV1-TV6) showed low or no expression in various tissues overall (Fig. 2d). We further investigated ACTR8 gene expression of other primates (humans, rhesus monkey, African green monkey, marmoset, and squirrel monkey) using cerebellum cDNA samples and transcript variant-specific primers (Fig. 2e). In humans and Old world monkeys, transcript variants showed the same expression patterns as those of the crab-eating monkey. Moreover, each transcript variant appeared to have a similar expression level to that of the crab-eating monkey. Remarkably, New world monkeys only expressed the original transcript. Our data suggest that the expression of the ACTR8 gene is regulated by lineage-specific AS events in primates. 


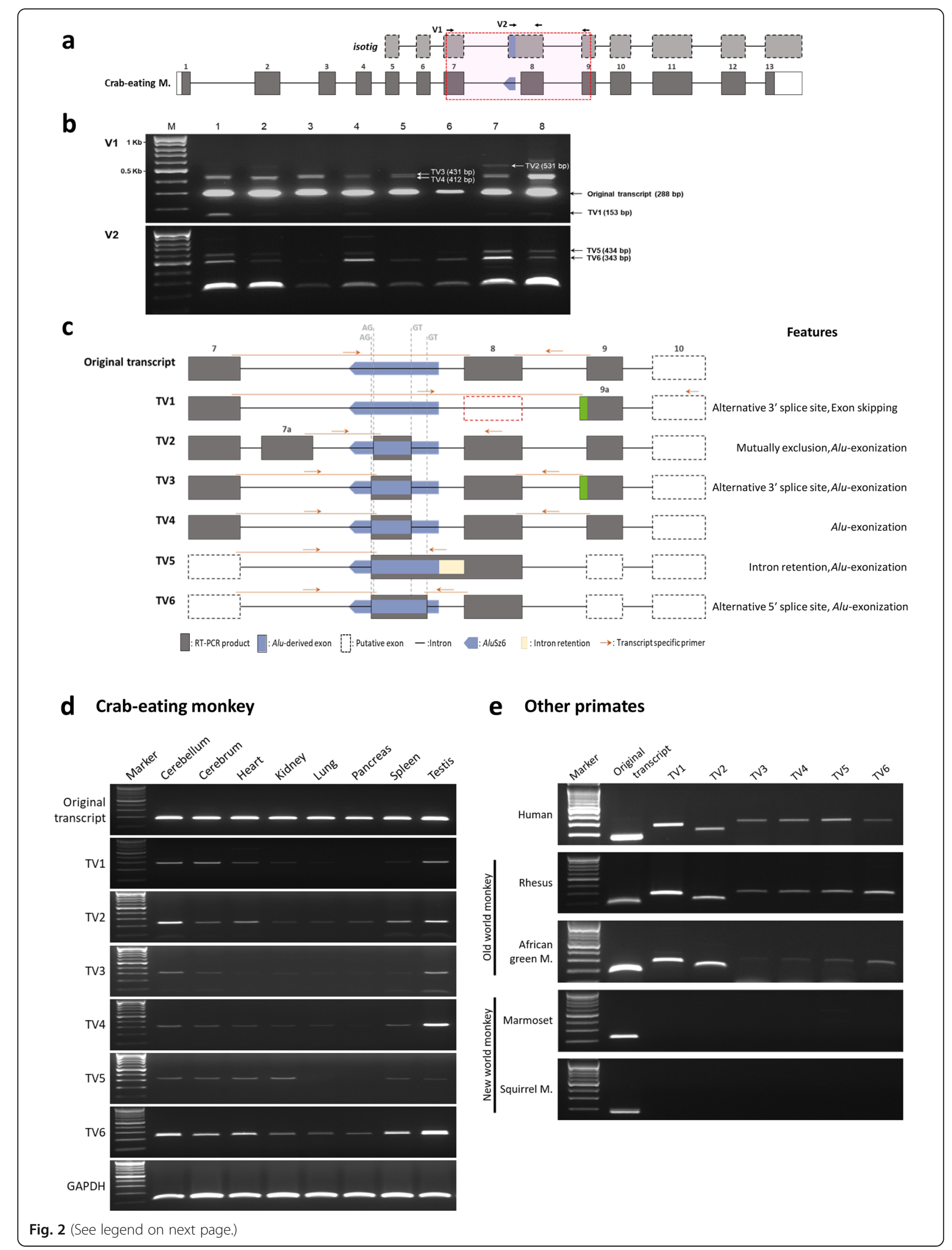


(See figure on previous page.)

Fig. 2 ACTR8 gene transcript variants in primates using RT-PCR and sequence analysis. a The AluSz6 element-integrated transcript was validated using the V1 and V2 primer pairs. Grey and dotted boxes represent the coding exon and putative exon, respectively. Horizontal arrows indicate the V1 and V2 primer location. $\mathbf{b}$ RT-PCR was performed using the tissue CDNA of the crab-eating monkey, and the PCR product was electrophoresed by agarose gel electrophoresis and stained with ethidium bromide. Seven positive bands were observed: 1 , cerebellum; 2 , cerebrum; 3, heart; 4, kidney; 5, lung; 6, pancreas; 7, spleen; and 8, testis c Seven transcript variants were identified. Grey boxes represent the coding exon and PCR product. Vertical dashed lines represent the $3^{\prime}(\mathrm{AG})$ and $5^{\prime}(\mathrm{GT})$ splicing sites. The green box indicates the exon $9 \mathrm{a}$, which is longer than exon 9. The orange-colored arrows represent the validation of RT-PCR primers of each transcript variant. $\mathbf{d}$ In the crab-eating monkey, RT-PCR was performed using various tissues and specific primer pairs. GAPDH (120 bp) indicates the positive control. e Human, Old world monkey (rhesus monkey, African green monkey), and New world monkey (marmoset, squirrel monkey) cerebellar cDNA were used for the validation of the transcript variant expression

\section{Bioinformatics analysis of alternative splicing transcripts} Multiple sequence alignment of the AluSz6-derived exon in nine primates (humans, chimpanzee, gorilla, rhesus monkey, African green monkey, marmoset, squirrel monkey, and ring-tailed lemur) demonstrated that a novel $\mathrm{G}$ duplication was used as the new $5^{\prime}$ SS for the AluSz6-derived exon in Old world monkeys and apes (Fig. 3 and Additional file 1: Figure S1A). Considering our results, lineage-specific $A l u$ exonization in the ACTR8 gene might be caused by the $\mathrm{G}$ duplication mutation, which is absent in New world monkeys and prosimians. We carefully proved our findings that difference AluSz6 sequences among the primates, matching with AluSz6 sequences from the UCSC genome browser. We found a discrepant $\mathrm{G}$ duplication in the AluSz6 sequence in primate lineages (Additional file 1: Figure S4).

Moreover, we identified lineage-specific exon inclusion or exclusion events, regardless of AluSz6 integration. The TV2 transcript carrying exon 7 a showed lineagespecific expression. The splice sites (donor and acceptor site) were well conserved in all primates evaluated (Additional file 1: Figure S1). Assessment of the branch point using the SVM-BP finder to the surrounding sequences of exon 7a predicted several branch point candidates. Among the candidates, the TTATAAGAT sequence had the highest potential for inclusion in exon $7 \mathrm{a}$. This sequence was located $21 \mathrm{bp}$ upstream of the 3 ' SS of exon 7a in Old world monkeys and apes, but not New world monkeys and prosimians (Additional file 1: Figure S1). It is likely that a lineage-specific mutual exclusion exon, exon 7a, may have been spliced due to the branch point difference (Additional file 1: Figure S1). In the squirrel monkey, exons 2 and 3 were found to be longer than in the other primates (Fig. 1a). We examined the splice site of the constitutive exon. Interestingly, the squirrel monkey has a TA sequence, a specific acceptor splice site, whereas Old world monkeys and apes do not have the 5' SS consensus sequence in the same region (Additional file 1: Figure S2). The TA acceptor splice site is also seen in marmosets and lemurs, and although they are likely to express the longer exons 2 and 3, we did not confirm their expression experimentally (Additional file 1 :
Figure S2). Therefore, besides AluSz6, factors promote lineage-specific expression in the primate ACTR 8 gene.

\section{Analysis of ACTR8 isoform structure and function}

To assess how the seven transcript variants identified affect translation and protein function, we performed in silico analysis using the ORF finder (http://www.ncbi. nlm.nih.gov/projects/gorf/) and Pfam (https://pfam.xfam. org) database. In this study, seven transcript variants produced a total of four isoforms of the ACTR8 protein, containing the original and alternative isoforms. The original transcript encodes the full-length protein with 624 amino acids, including an ATP-binding site (amino acids (aa) 55-56, 288, and 290) and a nucleotide-binding site (aa 283-286) [37, 38]. The TV1 transcript encodes isoform 1 with 579 aa, deleting aa 308 to 352 . TV2 encodes isoform 2 with 341 aa, and TV3-TV6 encode isoform 3 with 304 aa (Fig. 4). Exon $7 \mathrm{a}$ and AluSz6 introduce a pre-termination codon (PTC) in TV2-TV6, and we identified truncated proteins, with isoforms 2 and 3 deleted from the C-terminus (Additional file 1: Figure S3). Notably, isoforms 2 and 3 certainly contained the ATPbinding pocket and nucleotide-binding sites, which are essential functional domains for correct ACTR8 functioning. According to a previous study, the N-terminal region of ACTR8 is critical for protein functional activity. N-terminal deletions have deleterious effects on the expressed protein [39-41]. However, deletions in the Cterminal region did not lead to defects in ACTR8 function [37]. Although the Alu-derived transcript in the ACTR8 gene altered the protein structure, obtained novel isoforms can produce a lineage-specific protein without loss of function.

\section{Discussion}

Alu elements belong to the SINE family of TEs and are primate-specific repetitive elements. In humans, they make up $11 \%$ of the genome. Alu elements emerged 65 million years ago, before the radiation of primates, by a fusion of the $5^{\prime}$ and $3^{\prime}$ ends of the 7 SL RNA gene [42, 43]. Once an $\mathrm{Alu}$ integrated at a chromosome locus, it might have copied itself for transposition. Thus, its copy 


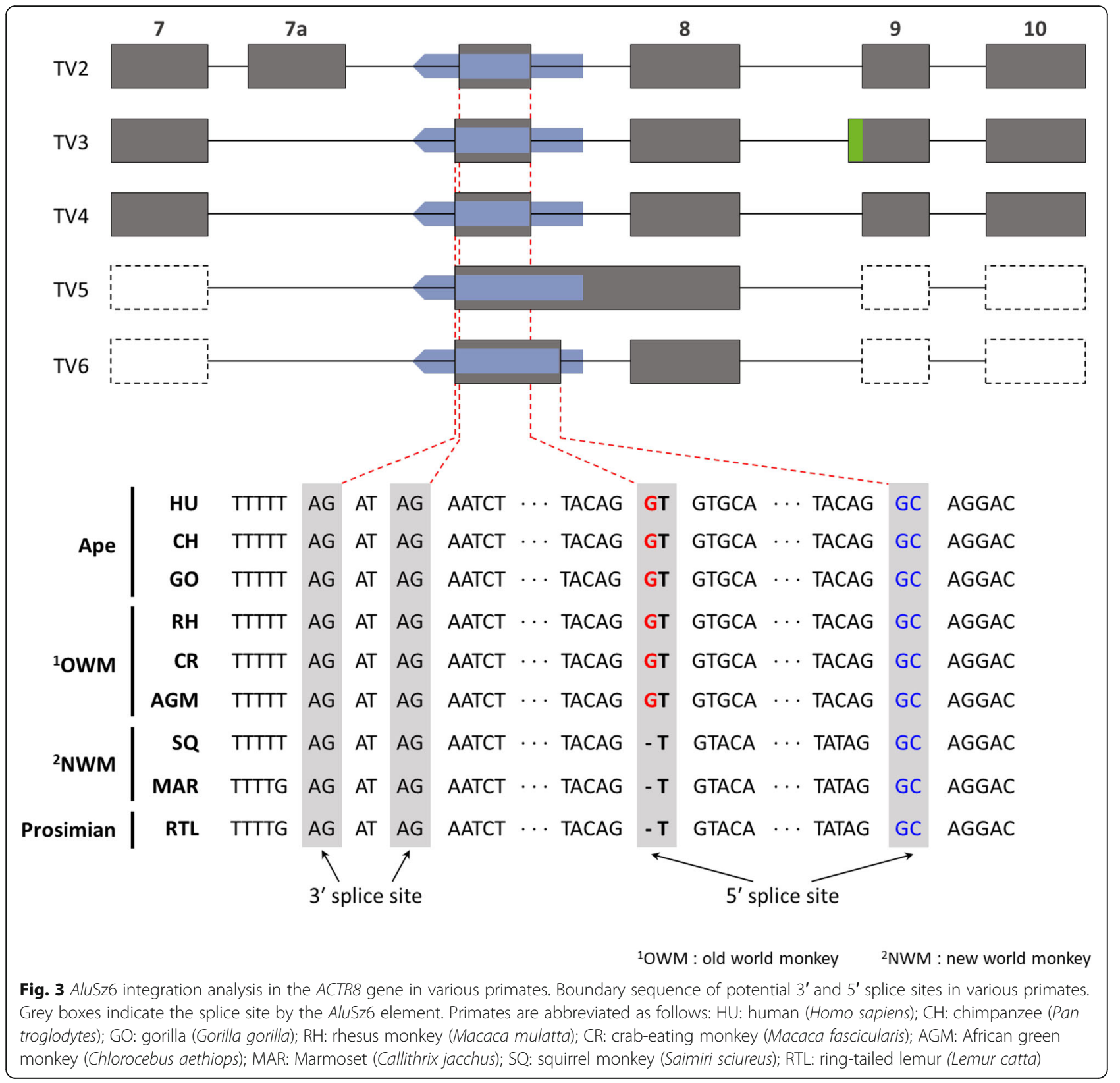

numbers increased throughout primate evolution [19, 44]. Although Alu elements inserted in non-coding regions are thought to be evolutionarily neutral, many scientists have demonstrated that TEs evidently play a key role in primate evolution by creating new mutations and gene combinations [45]. Alu elements integrated into the human genome sometimes have little effect on the phenotype [43], but $A l u$ elements created a significant increase in transcriptome diversity and contributed to unique features of primates [19, 44, 45]. The integration time of locusspecific Alu elements can indicate evolutionary time in primate lineages. Alu elements are useful tools to study genome evolution, phylogenetics, and population biology in primates $[45,46]$. Moreover, Alu-exonized transcripts can be useful for analyzing the mechanism underlying the creation of new transcript variants during primate evolution by estimating the transcript generation time [47-49].

Alu elements inserted in the human genome are distributed to introns in both $55 \%$ antisense and $45 \%$ sense orientations [50]. The sense-orientation Alu elements provide splicing silencer sequences that repress their exonization [51], while antisense oriented $\mathrm{Alu}$-elements contain strong PPT and many potential $5^{\prime}$ and $3^{\prime}$ splice sites and therefore tend to be alternatively spliced at a higher frequency than the original exon $[24,50]$. Alu elements can be divided into distinct lineages or families 


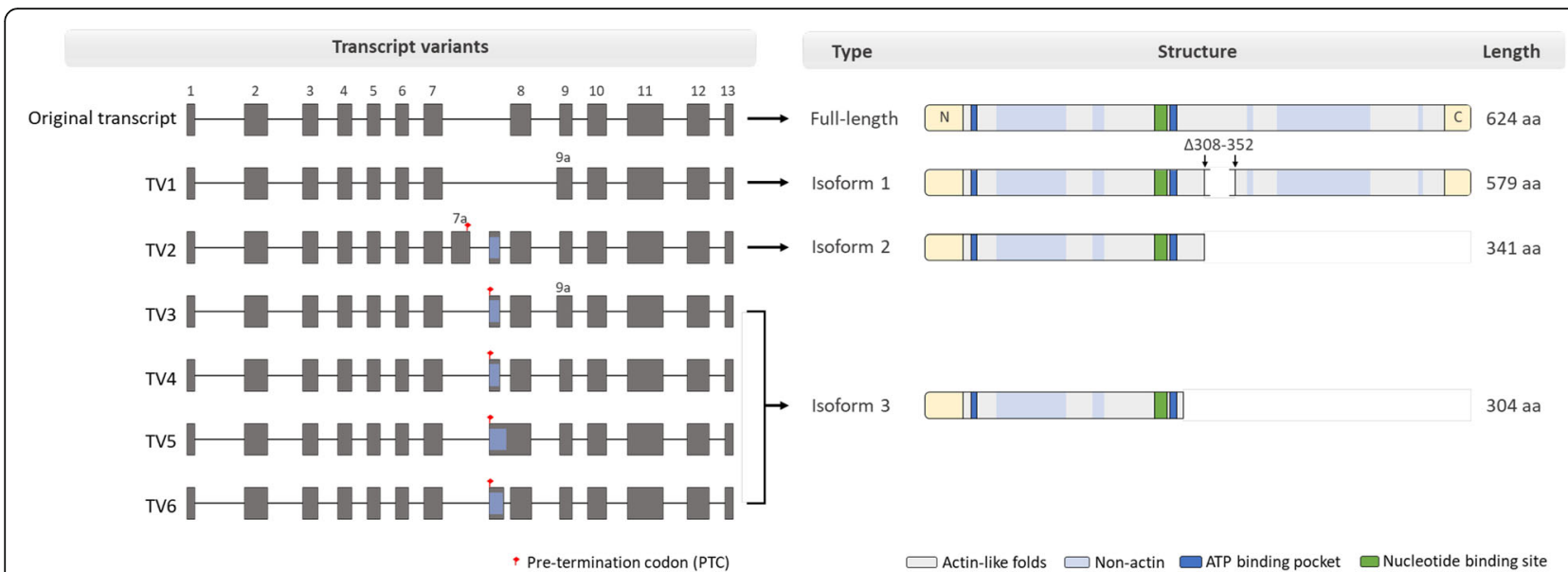

Fig. 4 Schematic of the genomic and protein structures of the ACTR8 gene. Full-length ACTR8 of humans and crab-eating monkeys encoding 624 amino acids containing the actin, ATP-binding, and nucleotide-binding sites. The identified transcript variants encoded isoform 1 and isoforms $2-$ 3 lacking the C-terminal

based on inheritance patterns of new mutations [52]. The AluS subfamily, to which AluSz6 belongs, is the second oldest Alu lineage and $~ 30$ million years old, and it comprises 551,383 full-length copies in humans [53]. The AluS subfamily has recently been reported to be active in humans [54], and editing events such as A to I RNA editing occurs with high frequency in this subfamily [55]. Furthermore, Alu-mediated unequal recombination has been reported, resulting in mutation or polymorphic substitution, implying contribution to structure variation and $\mathrm{Alu}$ evolution [56, 57]. In addition, insertion of Alu elements might cause genetic disease and cancer through structural variation and genomic instability [58-61]. In fact, partial AluSz6 insertion in the ACAT1 gene has a negative effect downstream of exon inclusion, depending on the distance between the Alu element and the splice site acceptor site [62].

This study aimed to evaluate the AluSz6 integration event in the ACTR8 gene. Here, we discovered for the first time that AluSz6 has existed in a dormant state as junk DNA in the ACTR8 gene, and it was activated in the ACTR 8 gene in Old world monkey and ape lineages by a small mutation: $\mathrm{G}$ duplication (Fig. 3). We tried to rationalize our result that lineage-specific expression is caused by $\mathrm{G}$ duplication in AluSz6. First, we validated our results based on findings from compiled data about Alu exonization in humans [63]. Lev-Maor et al. showed that an inverted $A l u$ insertion sequence used a $3^{\prime}$ SS, proximal AG, and this is especially well shown in the AluS subfamily. Our results indicated that the use of proximal AG was preferred over distal AG, similar to the findings of Lev-Maor et al. [63]. Second, we suggest the molecular mechanism of the $\mathrm{G}$ duplication event in the AluSz6 element. Two molecular mechanisms might explain our results: there might be secondary unequal homologous recombination or DNA replication-derived errors $[60,64,65]$. Commonly, unequal homologous recombination is characterized by nearby Alu elements or L1 sequence in the genome and duplication or deletions of the segments [66]. We speculated that the single $\mathrm{G} \mathrm{du}$ plication in AluSz6 is the error that occurred during DNA replication. Therefore, we found that molecular mechanism about the occurrence of single mutation in AluSz6.

The original transcript ( $288 \mathrm{bp}$ ) was more strongly expressed compared to the other transcripts (153 bp, $412 \mathrm{bp}$, $431 \mathrm{bp}$, and $531 \mathrm{bp}$ ). The original transcript showed a 13.1fold higher intensity compared to the weakly expressed transcript TV4 (Additional file 1: Figure S5). These gaps in expression levels suggest that the original transcript is a constitutive transcript, and that the other transcript variants are the minor expressed forms. Moreover, in various tissues, other transcript variants except the original were either not expressed or very weakly expressed (Fig. 2d, e).

ACTR8 links to $\mathrm{H} 2 \mathrm{~A}$ via the $\mathrm{C}$-terminal, and the $\mathrm{N}$ terminal binds to the DNA. Recent studies have reported that C-terminal loss does not affect functional activity [37, 41]. Our newly identified transcript variants express a truncated protein with the deletion of the C-terminaltruncated yet functional protein, containing the two core domain sequences (Fig. 4). Thus, we posit that the novel ACTR8 isoforms caused by AluSz6 insertion do not lead to functional defects in the protein. There are only lineage-specific differences in primates. To understand the lineage-specific evolution of ACTR8, we identified and analyzed ACTR8 isoforms in some primates, as well as representative mammals (mouse, rat, dog, horse, cow, and pig) (Fig. 5). Schematic comparison among the 15 mammals was performed on the basis of registered protein information in NCBI resources. Furthermore, the results of the experimental validation from primates were added to the protein comparison sets. As expected, 


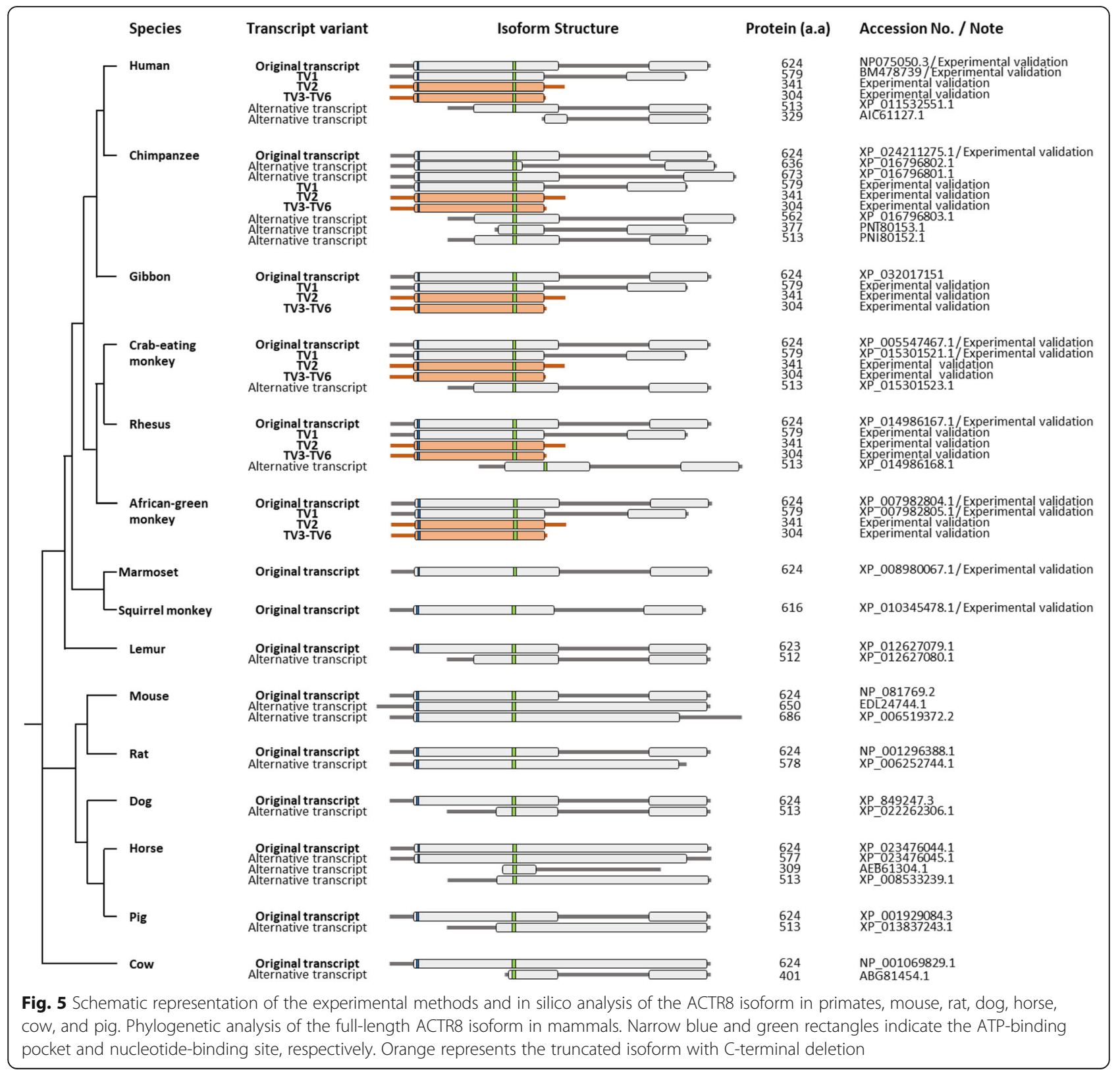

full-length isoforms were highly conserved in all tested mammals with 624 amino acids. Intriguingly, apes and Old world monkeys expressed isoforms comprising the skipping transcript (TV1) and C-terminal deletion isoform by AluSz6 integration. Besides, $\mathrm{N}$-terminal-deleted isoforms were registered in the protein database of NCBI for human, chimpanzee, crab-eating monkey, rhesus monkey, lemur, dog, pig, and cow. Nevertheless, apes and Old world monkeys were able to generate truncated isoforms without being affected by the loss of ACTR8 function. Non-primate mammals did not produce the Cterminal deletion isoform owing to the absence of the integration of TE, particularly primate-specific TE. There is an appearance of primate-specific ACTR8 proteins. Consequently, these results were meaningful in that primates, including humans, may just get a chance for genomic diversity enhancement in a way that only primates could do through Alu element.

Taken together, our findings indicate that the AluSz6 integration event occurred prior to primate radiation. Also, the single G duplication in AluSz6 provided mechanism to production of the Alu-derived exon and presented in ACTR8 gene of apes and Old world monkeys (Fig. 6). At the same time, Old world monkeys and apes 


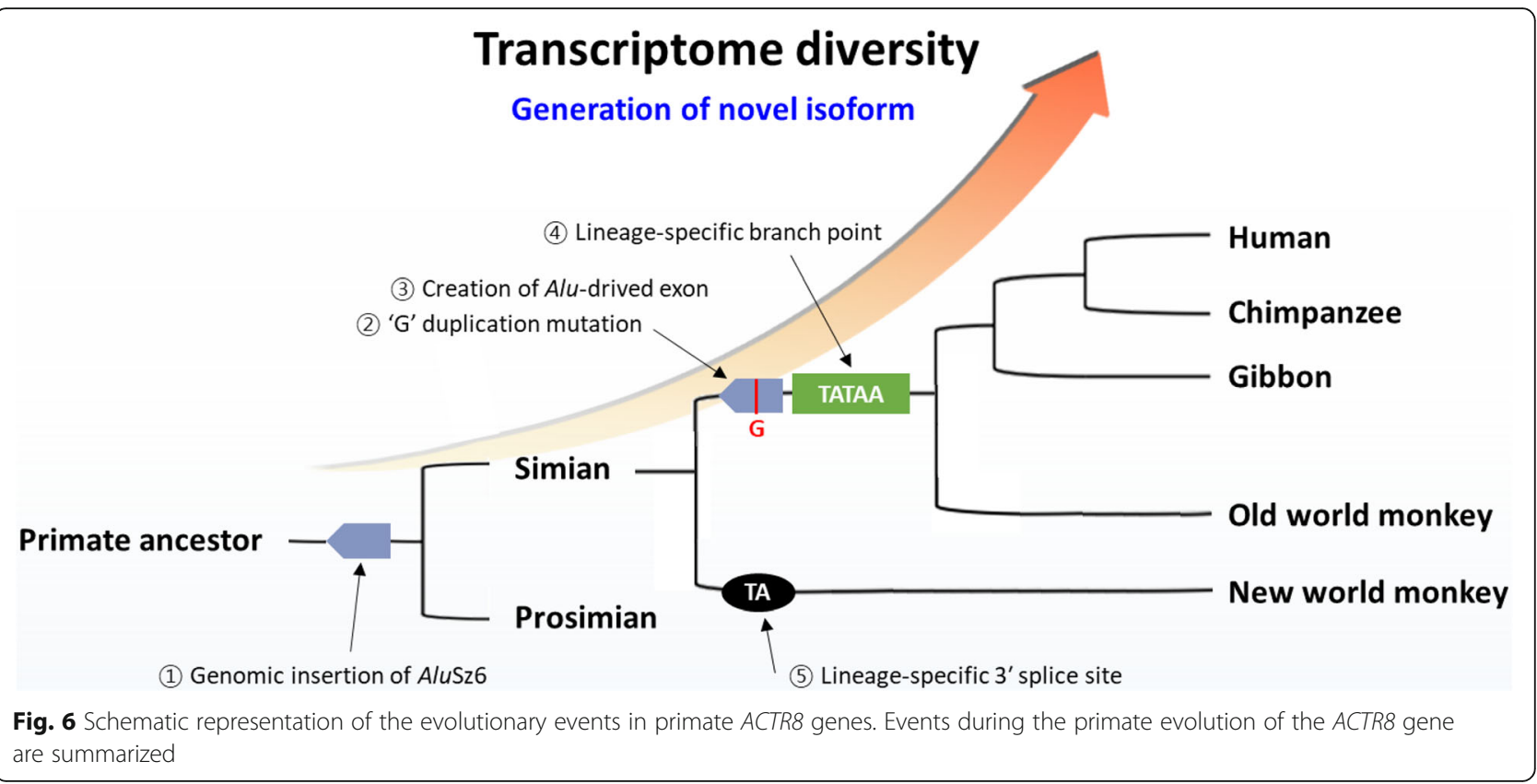

acquired a lineage-specific branch-point of the TATATAAGAT sequence. In addition, we characterized the unique splice donor site in New world monkeys following the lineage-specific transcript. Therefore, not only a single duplication in AluSz6, but also other lineage-specific genomic features appear to be concerned with lineage-specific expression and transcriptomic diversity, which may eventually play an important role in primate evolution.

\section{Conclusions}

The current study revealed the step-by-step evolutionary events in the ACTR8 gene that contributed to transcriptome diversity and the generation of novel isoforms of this gene in primates.

\section{Methods}

Total RNA and genomic DNA extraction

The total RNA samples isolated from different human tissues, including the cerebellum, cerebrum, heart, kidney, lung, liver, spleen, and testis, were purchased from Clontech Laboratories, Inc. The total RNA of nonhuman primates was extracted from the following tissues of the following animals: crab-eating monkey (cerebellum, cerebrum, heart, kidney, lung, pancreas, spleen, and testis), African green monkey (cerebrum), and common marmoset (cerebrum). The RNA samples were isolated using the RNeasy ${ }^{\circ}$ Plus Mini kit (Qiagen), according to the manufacturer's instructions. DNA contamination was eliminated by using the genomic DNA eliminator column and RNase-free DNase (Qiagen). The RNA concentration and purity (A260/A280) were determined with the ND-1000 spectrophotometer (NanoDrop Technologies), and the integrity of RNA was further confirmed by running the samples on an agarose gel. About 500 ng of total RNA was used for complementary DNA synthesis using the GoScript Reverse Transcription System (Promega) according to the manufacturer's instructions. The primate genomic DNA samples (HU: Homo sapiens, CH: Pan troglodytes, GO: Gorilla gorilla, RH: Macaca mulatta, CR: Macaca fascicularis, AGM: Cercopithecus aethiops, MAR: Callithrix jacchus, SQ: Saimiri sciureus, RTL: Lemur catta) and all the non-human primate samples were provided by the National Primate Research Center (NPRC) of Korea.

\section{RT-PCR and genomic PCR}

ACTR8 transcripts were analyzed by RT-PCR amplification using the following primer pairs: V1 (S: 5'-AGG AGT CTG TGT GTG CCA-3', AS: 5'-GAA GCC CAG AGA TGT CCT GA-3'); V2 (S: 5'-TTT AAG GTG TTG CAG AAG AAG AC-3', AS: 5'-TTT AAG GTG TTG CAG AAG AAG AC-3'). RT-PCR was conducted using the ExPrime Taq premix (Genetbio) under the following conditions: pre-denaturation for $5 \mathrm{~min} ; 30 \sim 35$ cycles at $94{ }^{\circ} \mathrm{C}$ for $30 \mathrm{~s}, 57-61{ }^{\circ} \mathrm{C}$ for $30 \mathrm{~s}, 72^{\circ} \mathrm{C}$ for $30 \sim$ $40 \mathrm{~s}$; and a final elongation at $72^{\circ} \mathrm{C}$ for $5 \mathrm{~min}$. To analyze the integration time of the AluSz6 element during primate evolution, genomic DNAs from different primate lineages were amplified using specific primer sets designed from well-conserved regions (S: 5'-TAT GCT GTG TGG AGG ATG GG-3', AS: 5' -CTG GCA TTC TCT GTA AGG GAA C-3'). The genomic PCR was carried out under the following conditions: $94{ }^{\circ} \mathrm{C}$ for $5 \mathrm{~min}$; 
30 cycles at $94{ }^{\circ} \mathrm{C}$ for $30 \mathrm{~s}$, at $56{ }^{\circ} \mathrm{C}$ for $30 \mathrm{~s}$, at $72{ }^{\circ} \mathrm{C}$ for $90 \mathrm{~s}$; and a final elongation step at $72{ }^{\circ} \mathrm{C}$ for $5 \mathrm{~min}$. The GAPDH gene was used as a standard control and was analyzed using specific primer pairs (S: 5' - GAA ATC CCA TCA CCA TCT TCC AGG-3', AS: 5' - GAG CCC CAG CCT TCT CCA TG- 3') designed based on the human GAPDH sequence.

\section{Molecular cloning and sequencing}

For the validation and sequencing of the PCR products, all PCR products were separated on a $1.5 \%$ agarose gel, purified with the Gel SV Extraction kit (GeneAll), and cloned into the RBC T\&A Cloning Vector. The cloned DNA was isolated using the Plasmid DNA Mini-prep kit (GeneAll) and sequenced by Macrogen. The validation of primate DNA samples and alternative transcripts was performed as mentioned above.

\section{Branch-point analysis}

Splicing is catalyzed by the spliceosome, a large RNAprotein complex, and this complex binds to the branch point site, which is a short sequence upstream of the acceptor splice sites ( $3^{\prime}$ SS). As the branch point sequences are associated with the cryptic and canonical 3' SSs, branch-point prediction is important in pre-mRNA splicing. The SVM-BPfinder program (http://regulatorygenomics.upf.edu/Software/SVM_BP/) [67] was used to analyze the branch point site in the 7 th intron sequence of the ACTR8 gene.

\section{Comparative in silico analysis of the ACTR8 protein in different species}

All ACTR8 protein sequences were collected from the NCBI database in FASTA format for the following different species: human, chimpanzee, gibbon, crab-eating monkey, rhesus monkey, African green monkey, marmoset, rat, dog, horse, cow, and pig. The BioEdit program was used for multiple sequence comparison. Pairwise alignments were used to determine the identity and similarity between each species. On the basis of the fulllength sequence of the ACTR8 protein, the phylogeny tree of the ACTR8 protein was obtained by the BioEdit and TreeView programs.

\section{Acquisition and quantitative analysis of PCR-gel images}

The separated PCR products on the ethidium bromide-stained gels were scanned using the gel image software (Vision-capt, Vilber). Band intensity was calculated by the volume, based on the height and area of the positive bands, and the relative intensity (\%) was determined.

\section{Supplementary information}

Supplementary information accompanies this paper at https://doi.org/10. 1186/s12862-020-01620-9.

Additional file 1: Table S1. List of primers used in this study. Figure S1 Multiple sequence alignment of the AluSz6 insertion region in the ACTR8 gene in various primates. Sequences obtained from the genomic PCR product (Fig. 1b). Black lined boxes indicate exons, and sequences between boxes are intron regions. Two forward slashes indicate the region of omitted intron sequence for good-quality data. The sky-blue region represents the full length of AluSz6. 3' Splice sites and 5' splice sites in the AluSz6 region are not in sky blue. The black-shaded region indicates the branch point site, and New world monkeys and prosimians have a deletion in this branch point region. Figure S2 Multiple sequence alignment of the region from exon 2 to exon 4 in the ACTR8 gene in various primates. Genomic sequences from the NCBI database were aligned in various primates. The donor splice site (5' splice site) and acceptor splice site ( $3^{\prime}$ splice site) are indicated as blue and red dotted line boxes, respectively. Figure $\mathbf{S 3}$ Multiple sequence alignment of the RT-PCR products using the validation primer. The RT-PCR products were sequenced, and these sequences were analyzed by multiple nucleotide alignment. The sky-blue region indicates the AluSz6-derived exon region. The red lined box represents the premature termination codon (PTC). Figure S4 Multiple sequence alignment of AluSz6 of the ACTR8 gene. AluSz6 sequences of various primates were searched using online resources (UCSC Genome browser). In this figure, the AluSz6 sequences are shown in the forward direction ( $5^{\prime}$ to $\left.3^{\prime}\right)$, whereas the main figure is shown in the reverse direction as inserted into the genome. The blue lined box indicates the point mentioned as a G duplication. AluSz6 of RTL could not be analyzed, but we confirmed the AluSz6 sequence of RTL through the experiment. Figure S5 Analysis of the ACTR8 gene PCR band intensity. (A) Green lanes (1-5) were PCR-positive bands by the V1 primer from Fig. 2b. Vision-capt software selected five bands and generated peaks of the corresponding PCR band. The PCR band intensity was generated from these peaks. (B) Vision-capt software measured the volume based on height and area of the positive bands, respectively.

\begin{abstract}
Abbreviations
AS: Alternative splicing; Arps: Actin-related proteins; bp: Base pairs; ERVs: Endogenous retroviruses; LINEs: Long interspersed elements; ORF: Open reading frame; PTC: Premature termination codon; SINEs: Short interspersed elements; 3' SS: 3' splice site; TE: Transposable elements; UTR: Untranslated region; VNTRs: Variable number of tandem repeats; PPT: Polypyrimidine tract
\end{abstract}

\section{Acknowledgements}

Not applicable.

\section{Authors' contributions}

SHC, SJP, HMC, HRP, and JRL conducted all research procedures and analyses in the study, and SHC wrote the manuscript. SHC, SJP, and HMC contributed equally to this work. SHC and JWH designed the project. SHC, SJP, and JRL performed the experiments. SHC, HMC, HRP, and JRL analyzed the data. YHK and JWH supervised the research. SHC drafted the whole manuscript. SHC, SJP, HMC, YHK, and JWH contributed to the interpretation of the data and the revision of the manuscript. All authors read and approved the final manuscript.

\section{Funding}

This research was supported by a grant from the KRIBB Research Initiative program (KGM4622013, KGM5282022). The funders had no role in study design, collection, analysis, and interpretation of data and decision to publish, or preparation of the manuscript.

\section{Availability of data and materials}

All figures and tables generated in this study are available in this article and its additional files. 


\section{Ethics approval and consent to participate}

All the animal procedures were conducted based on the guidelines of the Institutional Animal Care and Use Committee (KRIBB-AEC-18144) of the Korea Research Institute of Bioscience and Biotechnology (KRIBB).

\section{Consent for publication}

Not applicable.

\section{Competing interests}

Authors declare that they have no competing interests.

\section{Author details}

'National Primate Research Center, Korea Research Institute of Bioscience and Biotechnology (KRIBB), Cheongju 28116, Korea. ${ }^{2}$ Department of Functional Genomics, KRIBB School of Bioscience, Korea University of Science \& Technology (UST), Daejeon 34113, Korea. ${ }^{3}$ Primate Resource Center, Korea Research Institute of Bioscience and Biotechnology (KRIBB), Jeongeup 56216, Korea.

\section{Received: 4 February 2020 Accepted: 29 April 2020}

\section{Published online: 05 June 2020}

\section{References}

1. Keren H, Lev-Maor G, Ast G. Alternative splicing and evolution: diversification, exon definition and function. Nat Rev Genet. 2010;11(5):345-55.

2. Bush SJ, Chen L, Tovar-Corona JM, Urrutia AO. Alternative splicing and the evolution of phenotypic novelty. Philos Trans R Soc Lond B Biol Sci. 2017; 372:1713.

3. Lee C, Wang Q. Bioinformatics analysis of alternative splicing. Brief Bioinform. 2005;6(1):23-33.

4. Pan Q, Shai O, Lee LJ, Frey BJ, Blencowe BJ. Deep surveying of alternative splicing complexity in the human transcriptome by high-throughput sequencing. Nat Genet. 2008:40(12):1413-5.

5. Cartegni L, Chew SL, Krainer AR. Listening to silence and understanding nonsense: exonic mutations that affect splicing. Nat Rev Genet. 2002;3(4): 285-98.

6. EZ WL, Zhou J. Splicing and alternative splicing in rice and humans. BMB Rep. 2013;46(9):439-47.

7. Kim E, Goren A, Ast G. Alternative splicing: current perspectives. Bioessays. 2008;30(1):38-47.

8. Kelley DR, Hendrickson DG, Tenen D, Rinn JL. Transposable elements modulate human RNA abundance and splicing via specific RNA-protein interactions. Genome Biol. 2014;15(12):537.

9. Abascal F, Tress ML, Valencia A. Alternative splicing and co-option of transposable elements: the case of TMPO/LAP2alpha and ZNF451 in mammals. Bioinformatics. 2015;31(14):2257-61.

10. Alekseyenko AV, Kim N, Lee CJ. Global analysis of exon creation versus los and the role of alternative splicing in 17 vertebrate genomes. RNA. 2007; 13(5):661-70.

11. Huh JW, Kim YH, Park SJ, Kim DS, Lee SR, Kim KM, Jeong KJ, Kim JS, Song BS, Sim BW, et al. Large-scale transcriptome sequencing and gene analyses in the crab-eating macaque (Macaca fascicularis) for biomedical research. BMC Genomics. 2012:13:163.

12. Sultana $T$, Zamborlini A, Cristofari G, Lesage P. Integration site selection by retroviruses and transposable elements in eukaryotes. Nat Rev Genet. 2017; 18(5):292-308

13. Belyayev A. Bursts of transposable elements as an evolutionary driving force J Evol Biol. 2014;27(12):2573-84

14. Boeke JD, Garfinkel DJ, Styles CA, Fink GR. Ty elements transpose through an RNA intermediate. Cell. 1985;40(3):491-500.

15. Finnegan DJ. Eukaryotic transposable elements and genome evolution. Trends Genet. 1989;5(4):103-7.

16. Bourque G, Burns KH, Gehring M, Gorbunova V, Seluanov A, Hammell M, Imbeault M, Izsvak Z, Levin HL, Macfarlan TS, et al. Ten things you should know about transposable elements. Genome Biol. 2018;19(1):199.

17. Hedges DJ, Batzer MA. From the margins of the genome: mobile elements shape primate evolution. Bioessays. 2005;27(8):785-94.

18. Lander ES, Linton LM, Birren B, Nusbaum C, Zody MC, Baldwin J, Devon K, Dewar K, Doyle M, FitzHugh W, et al. Initial sequencing and analysis of the human genome. Nature. 2001:409(6822):860-921.

19. Deininger P. Alu elements: know the SINEs. Genome Biol. 2011;12(12):236.
20. Ule J. Alu elements: at the crossroads between disease and evolution Biochem Soc Trans. 2013:41(6):1532-5.

21. Chen LL, Yang L. ALUternative Regulation for Gene Expression. Trends Cell Biol. 2017;27(7):480-90.

22. Cordaux R, Batzer MA. The impact of retrotransposons on human genome evolution. Nat Rev Genet. 2009;10(10):691-703.

23. Sorek R, Lev-Maor G, Reznik M, Dagan T, Belinky F, Graur D, Ast G. Minimal conditions for exonization of intronic sequences: $5^{\prime}$ splice site formation in alu exons. Mol Cell. 2004;14(2):221-31.

24. Sorek R. The birth of new exons: mechanisms and evolutionary consequences. RNA. 2007;13(10):1603-8.

25. Muller J, Oma Y, Vallar L, Friederich E, Poch O, Winsor B. Sequence and comparative genomic analysis of actin-related proteins. Mol Biol Cell. 2005; 16(12):5736-48

26. Vieu E, Hernandez N. Actin's latest act: polymerizing to facilitate transcription? Nat Cell Biol. 2006;8(7):650-1.

27. Kandasamy MK, McKinney EC, Deal RB, Meagher RB. Arabidopsis ARP7 is an essential actin-related protein required for normal embryogenesis, plant architecture, and floral organ abscission. Plant Physiol. 2005:138(4):2019-32.

28. Dion V, Shimada K, Gasser SM. Actin-related proteins in the nucleus: life beyond chromatin remodelers. Curr Opin Cell Biol. 2010;22(3):383-91.

29. Kristo I, Bajusz I, Bajusz C, Borkuti P, Vilmos P. Actin, actin-binding proteins, and actin-related proteins in the nucleus. Histochem Cell Biol. 2016;145(4): 373-88.

30. Oma Y, Harata M. Actin-related proteins localized in the nucleus: from discovery to novel roles in nuclear organization. Nucleus. 2011;2(1):38-46.

31. Tosi A, Haas C, Herzog F, Gilmozzi A, Berninghausen O, Ungewickell C, Gerhold CB, Lakomek K, Aebersold R, Beckmann R, et al. Structure and subunit topology of the INO80 chromatin remodeler and its nucleosome complex. Cell. 2013;154(6):1207-19.

32. Chen $M$, Shen $X$. Nuclear actin and actin-related proteins in chromatin dynamics. Curr Opin Cell Biol. 2007;19(3):326-30.

33. Ford J, Odeyale $\mathrm{O}$, Shen $\mathrm{CH}$. Activator-dependent recruitment of SWI/SNF and INO80 during INO1 activation. Biochem Biophys Res Commun. 2008; 373(4):602-6.

34. Mersch B, Sela N, Ast G, Suhai S, Hotz-Wagenblatt A. SERpredict: detection of tissue- or tumor-specific isoforms generated through exonization of transposable elements. BMC Genet. 2007:8:78.

35. Lin L, Shen S, Tye A, Cai JJ, Jiang P, Davidson BL, Xing Y. Diverse splicing patterns of exonized Alu elements in human tissues. PLoS Genet. 2008:4(10): e1000225.

36. Tajnik M, Vigilante A, Braun S, Hanel H, Luscombe NM, Ule J, Zarnack K, Konig J. Intergenic Alu exonisation facilitates the evolution of tissue-specific transcript ends. Nucleic Acids Res. 2015;43(21):10492-505.

37. Saravanan M, Wuerges J, Bose D, McCormack EA, Cook NJ, Zhang X, Wigley DB. Interactions between the nucleosome histone core and Arp8 in the INO80 chromatin remodeling complex. Proc Natl Acad Sci U S A. 2012; 109(51):20883-8

38. Gerhold CB, Winkler DD, Lakomek K, Seifert FU, Fenn S, Kessler B, Witte G, Luger K, Hopfner KP. Structure of Actin-related protein 8 and its contribution to nucleosome binding. Nucleic Acids Res. 2012:40(21):1103646.

39. Brahma S, Ngubo M, Paul S, Udugama M, Bartholomew B. The Arp8 and Arp4 module acts as a DNA sensor controlling INO80 chromatin remodeling. Nat Commun. 2018:9(1):3309.

40. Aoyama N, Oka A, Kitayama K, Kurumizaka H, Harata M. The actin-related protein hArp8 accumulates on the mitotic chromosomes and functions in chromosome alignment. Exp Cell Res. 2008;314(4):859-68.

41. Osakabe A, Takahashi Y, Murakami H, Otawa K, Tachiwana H, Oma Y, Nishijima H, Shibahara Kl, Kurumizaka H, Harata M. DNA binding properties of the actin-related protein Arp8 and its role in DNA repair. PLoS One. 2014; 9(10):e108354.

42. Deininger PL, Jolly DJ, Rubin CM, Friedmann T, Schmid CW. Base sequence studies of 300 nucleotide renatured repeated human DNA clones. J Mo Biol. 1981;151(1):17-33

43. Hasler J, Strub K. Alu elements as regulators of gene expression. Nucleic Acids Res. 2006;34(19):5491-7.

44. Brookfield JF, Johnson $\amalg$. The evolution of mobile DNAs: when will transposons create phylogenies that look as if there is a master gene? Genetics. 2006:173(2):1115-23.

45. Deininger P. Alu Elements; 2006. p. 21-34 
46. Salem AH, Kilroy GE, Watkins WS, Jorde LB, Batzer MA. Recently integrated Alu elements and human genomic diversity. Mol Biol Evol. 2003;20(8):1349-61.

47. Ray DA, Batzer MA. Tracking Alu evolution in New World primates. BMC Evol Biol. 2005;5:51.

48. Kim YH, Choe SH, Song BS, Park SJ, Kim MJ, Park YH, Yoon SB, Lee Y, Jin YB, Sim BW, et al. Macaca specific exon creation event generates a novel ZKSCAN5 transcript. Gene. 2016;577(2):236-43.

49. Park SJ, Kim YH, Lee SR, Choe SH, Kim MJ, Kim SU, Kim JS, Sim BW, Song BS, Jeong KJ, et al. Gain of a New Exon by a Lineage-Specific Alu ElementIntegration Event in the BCS1L Gene during Primate Evolution. Mol Cells. 2015;38(11):950-8.

50. Sorek R, Ast G, Graur D. Alu-containing exons are alternatively spliced. Genome Res. 2002;12(7):1060-7.

51. Lei H, Vorechovsky I. Identification of splicing silencers and enhancers in sense Alus: a role for pseudoacceptors in splice site repression. Mol Cell Biol. 2005;25(16):6912-20.

52. Deininger PL, Batzer MA, Hutchison CA 3rd, Edgell MH. Master genes in mammalian repetitive DNA amplification. Trends Genet. 1992;8(9):307-11.

53. Bennett EA, Keller H, Mills RE, Schmidt S, Moran JV, Weichenrieder O, Devine SE. Active Alu retrotransposons in the human genome. Genome Res. 2008; 18(12):1875-83.

54. Mills RE, Bennett EA, Iskow RC, Devine SE. Which transposable elements are active in the human genome? Trends Genet. 2007;23(4):183-91.

55. Kim DD, Kim TT, Walsh T, Kobayashi Y, Matise TC, Buyske S, Gabriel A. Widespread RNA editing of embedded alu elements in the human transcriptome. Genome Res. 2004;14(9):1719-25.

56. Wildschutte JH, Baron A, Diroff NM, Kidd JM. Discovery and characterization of Alu repeat sequences via precise local read assembly. Nucleic Acids Res. 2015;43(21):10292-307.

57. Kryatova MS, Steranka JP, Burns KH, Payer LM. Insertion and deletion polymorphisms of the ancient AluS family in the human genome. Mob DNA. 2017;8:6.

58. Fazza AC, Sabino FC, de Setta N, Bordin NA Jr, da Silva EH, Carareto CM. Estimating genomic instability mediated by Alu retroelements in breast cancer. Genet Mol Biol. 2009;32(1):25-31.

59. Zhang W, Edwards A, Fan W, Deininger P, Zhang K. Alu distribution and mutation types of cancer genes. BMC Genomics. 2011;12:157.

60. Kim S, Cho CS, Han K, Lee J. Structural Variation of Alu Element and Human Disease. Genomics Inform. 2016;14(3):70-7.

61. Song X, Beck CR, Du R, Campbell IM, Coban-Akdemir Z, Gu S, Breman AM, Stankiewicz P, Ira G, Shaw CA, et al. Predicting human genes susceptible to genomic instability associated with Alu/Alu-mediated rearrangements. Genome Res. 2018;28(8):1228-42.

62. Nakama M, Otsuka H, Ago Y, Sasai H, Abdelkreem E, Aoyama Y, Fukao T. Intronic antisense Alu elements have a negative splicing effect on the inclusion of adjacent downstream exons. Gene. 2018;664:84-9.

63. Lev-Maor G, Sorek R, Shomron N, Ast G. The birth of an alternatively spliced exon: 3' splice-site selection in Alu exons. Science. 2003:300(5623):1288-91.

64. Khitrinskaia I, Stepanov VA, Puzyrev VP. Alu repeats in the human genome. Mol Biol (Mosk). 2003;37(3):382-91.

65. Chen L, Zhou W, Zhang L, Zhang F. Genome architecture and its roles in human copy number variation. Genomics Inform. 2014;12(4):136-44.

66. Aleshin A, Zhi D. Recombination-associated sequence homogenization of neighboring Alu elements: signature of nonallelic gene conversion. Mol Biol Evol. 2010;27(10):2300-11.

67. Corvelo A, Hallegger M, Smith CW, Eyras E. Genome-wide association between branch point properties and alternative splicing. PLoS Comput Biol. 2010;6(11):e1001016

\section{Publisher's Note}

Springer Nature remains neutral with regard to jurisdictional claims in published maps and institutional affiliations.

Ready to submit your research? Choose BMC and benefit from:
- fast, convenient online submission
- thorough peer review by experienced researchers in your field
- rapid publication on acceptance
- support for research data, including large and complex data types
- gold Open Access which fosters wider collaboration and increased citations
- maximum visibility for your research: over 100M website views per year
At BMC, research is always in progress.
Learn more biomedcentral.com/submissions

